\title{
Bacillus subtilis as heterologous host for the secretory production of the non-ribosomal cyclodepsipeptide enniatin
}

\author{
Sophia Zobel • Jana Kumpfmüller • \\ Roderich D. Süssmuth • Thomas Schweder
}

Received: 26 August 2014 / Revised: 28 October 2014 / Accepted: 29 October 2014 /Published online: 15 November 2014

(C) The Author(s) 2014. This article is published with open access at Springerlink.com

\begin{abstract}
The heterologous expression of genes or gene clusters in microbial hosts, followed by metabolic engineering of biosynthetic pathways, is key to access industrially and pharmaceutically relevant compounds in an economically affordable and sustainable manner. Therefore, platforms need to be developed, which provide tools for the controlled synthesis of bioactive compounds. The Gram-positive bacterium Bacillus subtilis is a promising candidate for such applications, as it is generally regarded as a safe production host, its physiology is well investigated and a variety of tools is available for its genetic manipulation. Furthermore, this industrially relevant bacterium provides a high secretory potential not only for enzymes but also for primary and secondary metabolites. In this study, we present the first heterologous expression of an eukaryotic non-ribosomal peptide synthetase gene (esyn) coding for the biosynthesis of the small molecule enniatin in B. subtilis. Enniatin is a pharmaceutically used cyclodepsipeptide for treatment of topical bacterial and fungal infections. We generated various enniatin-producing $B$. subtilis strains, allowing for either single chromosomal or plasmid-based multi-copy expression of the esyn cluster under the control of an acetoin-inducible promoter system. Optimization of cultivation conditions, combined with
\end{abstract}

Sophia Zobel and Jana Kumpfmüller contributed equally to this work.

Electronic supplementary material The online version of this article (doi:10.1007/s00253-014-6199-0) contains supplementary material, which is available to authorized users.

S. Zobel $\cdot$ R. D. Süssmuth $(\bowtie)$

Institut für Chemie, Technische Universität Berlin, Strasse des 17.

Juni 124, 10623 Berlin, Germany

e-mail: roderich.suessmuth@tu-berlin.de

J. Kumpfmüller $\cdot$ T. Schweder $(\triangle)$

Institut für Pharmazie, Ernst-Moritz-Arndt-Universität,

Felix-Hausdorff-Strasse 3, 17489 Greifswald, Germany

e-mail: schweder@uni-greifswald.de modifications of the genetic background and multi-copy plasmid-based esyn expression, resulted in a secretory production of enniatin B. This work presents $B$. subtilis as a suitable host for the expression of heterologous eukaryotic non-ribosomal peptide synthetases (NRPS) clusters.

Keywords Heterologous expression - Iterative NRPS · Cyclodepsipeptide $\cdot a c o A$ promoter $\cdot$ Acetoin $\cdot$ Metabolic engineering

\section{Introduction}

There is an increasing demand for new bioactive natural products, which can in many cases not be covered from natural sources (Koehn and Carter 2005). Hence, quite often, the development of fermentation processes using suitable heterologous hosts is necessary. In addition, there are many safety requirements for the industrial production of enzymes and drugs (especially for food and pharmaceutical products) which are regulated and controlled by the Food and Drug Administration (FDA) or the European Medicine Agency (EMA).

Bacillus subtilis, a Gram-positive, non-pathogenic strain with a generally recognized as safe (GRAS) status and qualified presumption of safety (QPS) certification can fulfil these requirements (Leuschner et al. 2010; Sietske de Boer and Diderichsen 1991). Unlike Gram-negative hosts such as Escherichia coli, which feature lipopolysaccharides on the outer cell membrane, $B$. subtilis lacks these endotoxins, which simplifies downstream processing (Petsch and Anspach 2000). Furthermore, using B. subtilis as an expression host is advantageous due to its natural ability to secrete peptides into the environment, e.g. to interact with plants or pathogens (Mongkolthanaruk 2012). It is especially noteworthy that B. subtilis, in contrast to E. coli, is a more prolific natural 
producer of various bioactive compounds originating from non-ribosomal peptide synthetases (NRPS) and polyketide synthases (PKS) (Stein 2005). These include the nonribosomally synthesized lipopeptides surfactin (Arima et al. 1968; Nakano et al. 1991) and plipastatin showing antimicrobial activity (Tsuge et al. 2007), as well as the PKS/NRPShybrid molecule bacillaene with antibacterial effects (Butcher et al. 2007; Chen et al. 2007; Patel et al. 1995). In addition, B. subtilis was already successfully used as surrogate host for the engineered biosynthesis of the peptide antibiotic bacitracin from Bacillus licheniformis (Eppelmann et al. 2001). For the above reasons, $B$. subtilis is virtually predestined for heterologous NRPS production.

In this study, the NRPS-derived cyclohexadepsipeptide enniatin was used (Fig. 1), which is produced in a nonribosomal fashion by various filamentous fungi such as Fusarium and Verticillium species (Süssmuth et al. 2011). These filamentous fungi are characterized by long fermentation times and are nearly insusceptible to standard procedures of genetic manipulations for production level enhancement or pathway engineering. Enniatin is well-known for a broad range of bioactivities, and it shows various antibacterial, insecticidal, antifungal, herbicidal, anthelmintic and anticancer activities (Dornetshuber et al. 2007; Kamyar et al. 2004; Kleinkauf and von Döhren 1990; Kouri et al. 2003; Pleiss et al. 1996; Süssmuth et al. 2011).

To date, 29 natural enniatin derivatives are known, which differ in their amino acid and $\alpha$-hydroxy-carboxylic acid composition. A mixture of enniatin A, B and C finds application as fusafungin $\left(\right.$ Locabiosol $\left.^{\circledR}\right)$, a drug for treatment of upper respiratory tract infections (URTI) which shows bacteriostatic and anti-inflammatory properties against several microorganisms involved in superinfections, like Streptococcus pneumoniae, Staphylococcus aureus, Haemophilus influenzae and Candida albicans (German-Fattal 1988; Karam-Sarkis et al. 1991; Lund et al. 2004).

Enniatin is synthesized by the enniatin synthetase (ESYN), a non-ribosomal peptide synthetase which assembles the hexadepsipeptide in an iterative manner. The 347-kDa multienzyme complex consists of two modules. Each module provides a functional unit involving a condensation (C) domain, an adenylation (A) domain and a peptidyl carrier protein (PCP) domain (Glinski et al. 2002; Pieper et al. 1995). The A domains recognize their L-configured amino acid and D- $\alpha$ hydroxy carboxylic acid substrate and activate them in an ATP-dependent manner by adenylation at the carboxy group to yield acyl monoadenylates. The activated substrates are then transferred to a PCP domain, posttranslationally modified with a CoA arm, which is the acceptor for thiolation with the substrate. This PCP domain is phosphopantheteinylated by a phosphopantetheine transferase (PPTase). Intermediates that are covalently bound to the phosphopantetheine prosthetic group of the PCP domain are subsequently presented to the $\mathrm{C}$ domain, located upstream or downstream, responsible for the formation of the corresponding ester or amide bond between two activated substrates (Billich and Zocher 1988). An intermediate step is $N$-methylation of the Lamino acid by a methylation domain (MT domain). Emerging dipeptidoles are then temporarily stored at a so-called waiting position operated by a third PCP domain until the next dipeptidole subunit is formed and can be loaded onto the last PCP domain forming a 4-mer peptidole (Süssmuth et al. 2011). Upon availability of the third and last dipeptidole, the synthesis is completed by a last condensation and a final cyclisation step which takes place to release the active cyclohexadepsipeptide.

In this work, we established the first heterologous expression of a fungal non-ribosomal peptide synthetase and synthesis of the corresponding peptide enniatin in B. subtilis. In this study, the esyn gene encoding the enniatin synthetase (ESYN) from Fusarium oxysporum (Zocher et al. 1982) was used as a model for the heterologous expression of a NRPS cluster in B. subtilis under control of a glucose-repressed and acetoin-inducible promoter system (Ali et al. 2001; Kabisch et al. 2013b; Silbersack et al. 2006). In order to optimize the enniatin production in this host, several cultivation conditions
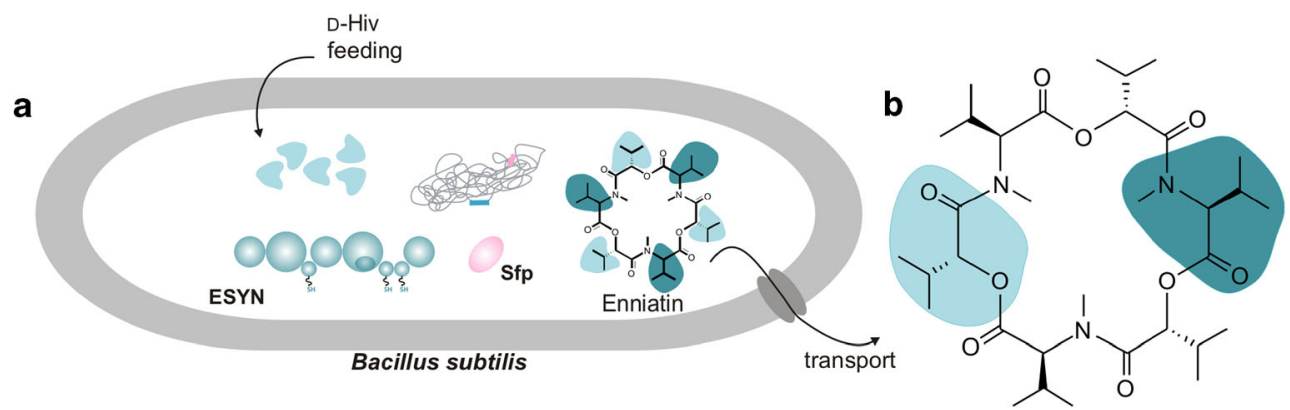

Fig. 1 Heterologous expression of enniatin in B. subtilis. a Integrative copy of esyn into the genome or a high-copy plasmid under the control of an acetoin-inducible promoter $(a c O A)$ combined with feeding of D-Hiv facilitates synthesis of enniatin. Enniatin is synthesized non-ribosomally by the ATP-dependent non-ribosomal peptide synthetase enniatin synthetase (ESYN). b Structure of the cyclohexadepsipeptide enniatin composed of alternating D-hydroxyisovalerate (D-Hiv) and L- $N$-methylvaline (L-N-Me-Val). The cyclic peptide is naturally produced by filamentous fungi of Fusarium spec 
and the deletion of potential competing gene clusters for native secondary metabolites were addressed.

\section{Materials and methods}

\section{Cloning}

Unless stated otherwise, all chemicals were purchased from Roth (Karlsruhe, Germany) at the highest purity available and were used without further purification. B. subtilis 168 (NCBI AL009126) was used as model production strain in this study. All cloning procedures were carried out in E. coli DH10B (Invitrogen, Darmstadt, Germany) [F-endA1 recA1 galE15 galK16 nupG rpsL $\Delta$ lacX74 $\Phi 80$ lacZ $\Delta$ M15 araD139 $\Delta$ (ara, leu) 7697 mcrA $\Delta$ (mrr-hsdRMS-mcrBC) $\lambda$-]. All plasmids used in this study are listed in Table 1. All strains used and constructed in this study are summarized in Table 2 . Restriction enzymes and other DNA-modifying enzymes were used as specified by the supplier (New England Biolabs, Frankfurt, Germany). Oligonucleotides (Table S1) were synthesized and provided by Life Technologies (Darmstadt, Germany). PCR products were purified with the High Pure PCR Product Purification Kit (Roche, Mannheim, Germany). Plasmid isolation was performed using the High Pure Plasmid Isolation Kit (Roche, Mannheim, Germany). For gel extraction, the QIAquick Gel Extraction Kit from Qiagen (Hilden, Germany) was used. Recombinant B. subtilis strains were verified by colony PCR as previously described (Kumpfmüller et al. 2013). All plasmid constructs and chromosomal integrations (s. supporting online material) were verified by sequencing carried out by Eurofins Genomics (Ebersberg, Germany).

\section{Media and cultivation}

For the cultivation of $B$. subtilis strains, a $20-\mathrm{mL}$ pre-culture was inoculated with a cryo-culture in LB medium $(10 \mathrm{~g} / \mathrm{L}$ tryptone, $5 \mathrm{~g} / \mathrm{L}$ yeast extract, $5 \mathrm{~g} / \mathrm{L} \mathrm{NaCl}$ ) supplemented with the corresponding antibiotic. After incubation for $16 \mathrm{~h}$ at $37^{\circ} \mathrm{C}$ in $50 \mathrm{~mL}$ super broth (SB) medium (containing $95 \%$ solution 1 buffered with $5 \%$ of solution 2 ; solution $1: 32 \mathrm{~g} / \mathrm{L}$ tryptone, $20 \mathrm{~g} / \mathrm{L}$ yeast extract, $5 \mathrm{~g} / \mathrm{L} \mathrm{NaCl}, \mathrm{pH} 7$; solution 2 : $12 \mathrm{~g} / \mathrm{L} \mathrm{Na} \mathrm{HPO}_{4}, 6 \mathrm{~g} / \mathrm{L} \mathrm{KH}_{2} \mathrm{PO}_{4}, 6 \mathrm{~g} / \mathrm{L} \mathrm{NH} \mathrm{NH}_{4} \mathrm{Cl}, 6 \mathrm{mg} / \mathrm{L}$ $\mathrm{CaCl}_{2}, \mathrm{pH} 7$ ), a second pre-culture was inoculated with 1:100 $(v / v)$ and $0.1 \%$ acetoin $(v / v)$ together with antibiotics and incubated at $37{ }^{\circ} \mathrm{C}$ to optical density at $600 \mathrm{~nm}$ $\left(\mathrm{OD}_{600 \mathrm{~nm}}\right)=1.0$. For the main culture, $100-\mathrm{mL}$ shaking flasks with three baffles were used containing $20 \mathrm{~mL}$ of SB medium supplemented with antibiotics and $0.1 \%$ acetoin $(v / v)$. The medium was inoculated at $\mathrm{OD}_{600 \mathrm{~nm}}=0.1$ and incubated for $1.5 \mathrm{~h}$ at $37^{\circ} \mathrm{C}$ to reach the exponential phase at $\mathrm{OD}_{600 \mathrm{~nm}}=0.6$. Subsequently, the cultures were fed with appropriate concentrations of $5 \mathrm{~mm} \mathrm{D}$-Hiv and incubated at $18{ }^{\circ} \mathrm{C}$ for $48 \mathrm{~h}$ under shaking conditions with $200 \mathrm{rpm}$.

\section{Extraction}

After cultivation and harvesting of cells from $20-\mathrm{mL}$ cultures, the supernatant was extracted with an equal volume of ethyl acetate and agitated for $1 \mathrm{~h}$ at room temperature. The cell pellet was extracted with $5 \mathrm{~mL}$ methanol and sonicated for $5 \mathrm{~min}$. After centrifugation, the solvent was removed and evaporated under vacuum. For mass spectrometric analysis, the extracts were dissolved in $200 \mu \mathrm{L}$ HPLC-grade $\mathrm{MeOH}$.

\section{HPLC-ESI-mass spectrometry}

All measurements for the analysis of crude extracts of pellet and supernatant were performed using an Agilent UHPLC 1290 Infinity-Series system containing an Eclipse Plus C18 column $(2.1 \times 50 \mathrm{~mm})$ coupled to an ESI-Triple-Quadrupol mass spectrometer (6460 Series, Agilent Technologies, Waldbronn, Germany). For chromatographic separation, a mobile phase $\mathrm{H}_{2} \mathrm{O}$ (solvent A)/ACN (solvent B) each with $0.1 \%$ formic acid $(v / v)$ was used. The gradient started at $5 \%$ solvent B to reach $100 \%$ in 4 min and was held constant for 3 min at $100 \%$ solvent B. For MS scan, $\mathrm{MS}^{2}$ and multiple reaction monitoring (MRM) analysis of enniatin with an exact mass $[\mathrm{M}]=639.4095 \mathrm{Da}$, all measurements were performed in the positive mode. The three most abundant fragments of $\mathrm{MS}^{2}$ experiments were used for quantification in MRM by fragmentation of precursor ion $\mathrm{m} / \mathrm{z} 640$ as well the characteristic mass transitions $\mathrm{m} / \mathrm{z} 527.4$ as quantifier and $\mathrm{m} / \mathrm{z} 427.3$ and $\mathrm{m} / \mathrm{z}$ 196.2 as qualifier ion. For relative quantification, we used a calibration curve of enniatin B and integrated the measured peak areas accordingly. Calibration curves comprising five concentrations in the range of $0.586-9.375 \mu \mathrm{g} / \mathrm{mL}$ were measured before and after the measurements of enniatin $\mathrm{B}$ from B. subtilis extracts (Fig. S1).

\section{Software}

Graphs were created using the graphical and statistical program R with the ggplot2 package (Wickham 2009). For box plots, the whiskers extend from the hinge to the highest and lowest value, respectively, that is within $1.5 \times \mathrm{IQR}$ of the hinge, where IQR is the inter-quartile range. Data beyond the end of the whiskers are outliers and plotted as points.

\section{Results}

Codon usage may exert an influence on gene expression (Nocon et al. 2014; Samant et al. 2014). Therefore, we first 
Table 1 Plasmids used in this study

\begin{tabular}{|c|c|c|}
\hline Plasmid & Function & Reference \\
\hline Fosmid F9D10 & Fosmid carrying wild-type esyn gene cluster & $\begin{array}{l}\text { Fosmid library of } F \text {. oxysporum ETH } 1536 \text {, } \\
\text { ESyn ACCN KP000028 }\end{array}$ \\
\hline pAMY-Kan & Backbone for $s r f A$ deletion plasmid with $\mathrm{Kan}^{\mathrm{R}}$ & Kumpfmüller, unpublished results \\
\hline pAMY-lox-SSS & Integration of genes into the $a m y E$ locus with lox-SSS-cassette & Kumpfmüller et al. 2013 \\
\hline pAMYSSE & Integration of genes into the $a m y E$ locus with SSE-cassette & Kabisch et al. 2013a \\
\hline pAMY-SSS & Integration of genes into the $a m y E$ locus with SSS-cassette & Kumpfmüller et al. 2013 \\
\hline pBB1366 & Integration of genes in $s a c A$ locus with $\mathrm{Cm}^{\mathrm{R}}$ & Middleton and Hofmeister 2004 \\
\hline pDGICZ & Source of cre operon and $\mathrm{Zeo}^{\mathrm{R}}$ & Yan et al. 2008 \\
\hline pJET-lox-SSS & Source of lox-SSS-cassette & Kumpfmüller et al. 2013 \\
\hline pJK45 & Integration of comS operon $\left(P_{\text {spac }}\right)$ into $s a c A$ locus with $\mathrm{Cm}^{\mathrm{R}}$ & This study \\
\hline pJK64 & Reconstitution of genetic $s f p$ defect with SSS-cassette & This study \\
\hline pJK64a & Reconstitution of genetic $s f p$ defect with lox-SSS-cassette & This study \\
\hline pJK93 & Deletion of $s r f A$ operon with remaining $\operatorname{Kan}^{\mathrm{R}}$ & This study \\
\hline pJK166 & Integration of $P_{a c o A^{-}}$esyn- $T_{T 7}$ into amyE locus & This study \\
\hline $\mathrm{pJK} 179$ & Deletion of $p k s X$ operon & This study \\
\hline pJK191 & Deletion of $s r f A$ operon & This study \\
\hline pJK195 & Integration of cre operon $\left(P_{x y l A}\right)$ into $s a c A$ locus with $\mathrm{Zeo}^{\mathrm{R}}$ & This study \\
\hline pJK196 & Integration of comS operon $\left(P_{\text {spac }}\right)$ and cre operon $\left(P_{x y l A}\right)$ into sacA locus with $\mathrm{Zeo}^{\mathrm{R}}$ & This study \\
\hline pJK205 & Insertion in $l y t C$ locus & This study \\
\hline pJK209 & Deletion of spoIIGA & This study \\
\hline pJK226 & Deletion of restriction and modification system (RM) & This study \\
\hline pJK210 & Integration of genes in $s a c A$ locus with $\operatorname{Spec}^{\mathrm{R}}$ & This study \\
\hline $\mathrm{pJK} 255$ & High-copy expression of $P_{a c o A^{-}}$esyn- $T_{T 7}$ & This study \\
\hline $\mathrm{pJK} 256$ & Substitution of comS operon $\left(P_{\text {spac }}\right)$ and cre operon $\left(P_{x y l A}\right)$ with $\mathrm{Spec}^{\mathrm{R}}$ & This study \\
\hline pKE19 & Source of $\operatorname{srfA} 5^{\prime}$-region with reconstituted comS gene & Eppelmann et al. 2001 \\
\hline pKE27 & Source of comS operon $\left(P_{\text {spac }}\right)$ & Eppelmann et al. 2001 \\
\hline pLytC & Backbone for $l y t C$ deletion plasmid with SSC-cassette & Kabisch et al. 2013b \\
\hline pMSE3 & High-copy E. coli/B. subtilis shuttle vector with $\operatorname{Kan}^{\mathrm{R}}$ & Silbersack et al. 2006 \\
\hline pSigL & Backbone for $p k s X$ deletion plasmid with SSE-cassette & Kabisch et al. $2013 b$ \\
\hline pSpoIIGA & Backbone for spoIIGA deletion plasmid with SSS-cassette & Kabisch et al. 2013b \\
\hline $\mathrm{pX}$ & Source of the $x y l A$-promoter and $x y l R$ gene & Kim et al. 1996 \\
\hline
\end{tabular}

$C m R$ chloramphenicol resistance cassette, EryR erythromycin resistance cassette, KanR kanamycin resistance cassette, SpecR spectinomycin resistance cassette, ZeoR Zeocin resistance cassette, ss six-site, lox 72 lox 72 site, SSS SpecR flanked by two ss, SSE EryR flanked by two ss, SSC CmR flanked by two ss, lox-SSS SSS surrounded by a lox71 and lox66 site

performed a bioinformatics analysis of the esyn gene. A comparison of the codon usage of the F. oxysporum esyn sequence with the average codon usage of dedicated host B. subtilis 168 showed a mean deviation of about $35 \%$ (Codon Usage Database, Kazusa; graphical codon usage analyser, Fig. S2). This analysis indicates that there is no significant codon bias which could influence the esyn expression in B. subtilis.

The plasmid construction for the chromosomal integration of the esyn gene, encoding the enniatin synthetase, was done via a modified protocol using the Red/ET recombination system (Zhang et al. 1998). Due to this method that is based on a crossover step between a targeting vector containing homologous regions and the target sequence (fosmid F9D10 carrying the natural esyn gene), amplification of the entire coding sequence (approx. $10 \mathrm{~kb}$ ) was not necessary. The resulting plasmid was designated pJK166. A detailed description of all plasmid and strain constructions can be found in the supplementary material.

The plasmid pJK166 was used to chromosomally integrate the esyn gene cluster under control of the $a c o A$ promoter into the $a m y E$ locus of $B$. subtilis JK3. This integration event was confirmed by colony PCR and an amylase-negative phenotype. Finally, one of the positive clones was chosen for marker removal (resulting in B. subtilis SZ2) followed by chromosomal integration of an intact $s f p$ gene by using the pJK64 
Table 2 Strains used in this study

\begin{tabular}{|c|c|c|}
\hline Strain & Relevant genotype & Reference \\
\hline B. subtilis 168 & Wild type, sfp $^{0}$ & Zeigler et al. 2008 \\
\hline B. subtilis ATCC 6051HGW & Wild type, sfp $^{+}$ & Kabisch et al. 2013b \\
\hline B. subtilis JK3 & $\Delta$ sacA $\because\left(\mathrm{Cm}^{R}, P_{\text {spac }}\right.$-comS, lacI $)$ & This study \\
\hline B. subtilis JK13 & $\Delta$ sacA $\because\left(Z_{e o}{ }^{R}, P_{\text {spac }}\right.$-comS, lacI, $P_{x y l A^{-}}$cre, xylR $)$ & This study \\
\hline B. subtilis JK28 & BsJK13+sfp ${ }^{+}: \because \operatorname{lox} 72$ & This study \\
\hline B. subtilis JK46 & BSJK28+RM::lox72 & This study \\
\hline B. subtilis JK75 & BsJK46+ $\Delta s r f A: \because \operatorname{lox} 72$ & This study \\
\hline B. subtilis JK76 & BsJK75+ $\Delta p k s X: \because \operatorname{lox} 72$ & This study \\
\hline B. subtilis JK77 & BsJK76+ $\Delta$ lytC $: \because \operatorname{lox} 72$ & This study \\
\hline B. subtilis JK78 & BsJK77+ $\Delta$ spoIIGA $: \because \operatorname{lox} 72$ & This study \\
\hline B. subtilis JK105 & BsJK78+ $\Delta$ amyE $:: \operatorname{lox} 72$ & This study \\
\hline B. subtilis JK106 & BsJK105+ $\Delta$ sacA $::$ Spec $^{R}$ & This study \\
\hline B. subtilis JK106 (pJK255) & High-copy expression strain & This study \\
\hline B. subtilis $\mathrm{SZ2}$ & BsJK3+ $\Delta a m y E::\left(P_{a c o A}-e s y n-T_{T 7}, s s\right)$ & This study \\
\hline B. subtilis $\mathrm{SZ4}$ & $B s S Z 2+s f p^{+}:: s s$ & This study \\
\hline B. subtilis $\mathrm{SZ5}$ & $B s S Z 4+\Delta s a c A: \because\left(Z e o^{R}, P_{s p a c}-c o m S\right.$, lacI, $P_{x y l A^{-}}$cre, xylR $)$ & This study \\
\hline B. subtilis $\mathrm{SZ6}$ & BsSZ5+ $\Delta l y t C: \because \operatorname{lox} 72$ & This study \\
\hline B. subtilis $\mathrm{SZ7}$ & BsSZ6+ $\Delta$ spoIIGA: :lox 72 & This study \\
\hline B. subtilis $\mathrm{SZ8}$ & $B s S Z 7+\Delta s a c A:: \operatorname{Spec}^{R}$ & This study \\
\hline B. subtilis $\mathrm{SZ9}$ & $B s S Z 7+\Delta s r f A: \because \operatorname{lox} 72$ & This study \\
\hline B. subtilis $\mathrm{SZ} 10$ & $B s S Z 9+\Delta s a c A: \because \operatorname{Spec}^{R}$ & This study \\
\hline B. subtilis SZ11 & 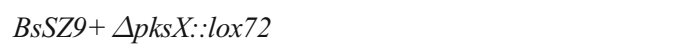 & This study \\
\hline B. subtilis $\mathrm{SZ} 12$ & $B s S Z 11+\Delta s a c A \because: S_{p e c}{ }^{R}$ & This study \\
\hline
\end{tabular}

$C m R$ chloramphenicol resistance cassette, SpecR spectinomycin resistance cassette, ZeoR Zeocin resistance cassette, ss six-site, lox 72 lox 72 site

plasmid. Obtained colonies were checked for haemolytic activity on sheep blood agar plates indicating restored surfactin production and hence PPTase activity due to a functional $s f p$ gene. After marker removal, one colony of the final strain B. subtilis SZ4 was chosen for genomic DNA isolation and the chromosomal reconstitution of the $s f p$ gene and the functionality of the esyn cluster were identified by means of PCR followed by DNA sequencing.

The selected esyn-encoding strain B. subtilis BsSZ4 was cultivated at $37^{\circ} \mathrm{C}$ in SB medium with $0.1 \%$ acetoin as the inducer. Supernatant and biomass were searched for the presence of enniatin (enniatin B is the main product of ESYN and, for reasons of simplicity, named enniatin) by means of HPLC-ESI-mass spectrometry. This analytics rendered the detection of enniatin adduct ions $[\mathrm{M}+\mathrm{H}]^{+}=640.4 \mathrm{Da},[\mathrm{M}+$ $\left.\mathrm{NH}_{4}\right]^{+}=657.4 \mathrm{Da}$ and $[\mathrm{M}+\mathrm{Na}]^{+}=662.4 \mathrm{Da}$ in the supernatant, whereas only minute amounts were found in the biomass (Fig. S3). Due to this repeated outcome of all fermentation runs, the subsequent analyses were mainly performed with culture supernatants.

The identity of enniatin was further corroborated by means of HPLC/ESI-MS ${ }^{2}$ experiments (Fig. S4) upon identification of characteristic masses of di- and tetrapeptidoles from fragmentation reactions at the less stable ester bonds. In the analytical assignment of secondary metabolites, we further observed the molecular masses of bacillaene and surfactin, previously identified from Bacillus species (Chen et al. 2007, Fig. S5). In MRM measurements, minor amounts of enniatin $(\sim 0.4 \mu \mathrm{g} / \mathrm{L})$ were detected.

In order to improve the enniatin production, variations in the cultivation conditions like temperature, concentration of the inductor acetoin and availability of required precursors in the form of D-hydroxyisovalerate (D-Hiv) and L-valine (L-Val) were tested. To minimize the metabolic burden for cells expressing a multi-enzyme of that size like enniatin synthetase (Pfeifer and Khosla 2001), we lowered the incubation temperature to slow down recombinant protein synthesis (Schein 1989). On the one hand, we chose $30{ }^{\circ} \mathrm{C}$ as well-known growth temperature of the ubiquitous soil bacterium B. subtilis. On the other hand, according to expression results, we defined $18^{\circ} \mathrm{C}$ as reasonable compromise between bacterial growth and protein expression. Remarkably, lowering the temperature resulted in higher enniatin synthesis (Fig. 2) compared to 37 or $30{ }^{\circ} \mathrm{C}$. Furthermore, also a doubling of the cultivation duration led to a significantly increased enniatin production. 
Fig. 2 Increased enniatin production by shifting the cultivation condition to lower temperatures. Cultures of BsSZ4 were grown under the same conditions with different temperatures $\left(18,30\right.$ and $\left.37^{\circ} \mathrm{C}\right)$. $N=4$

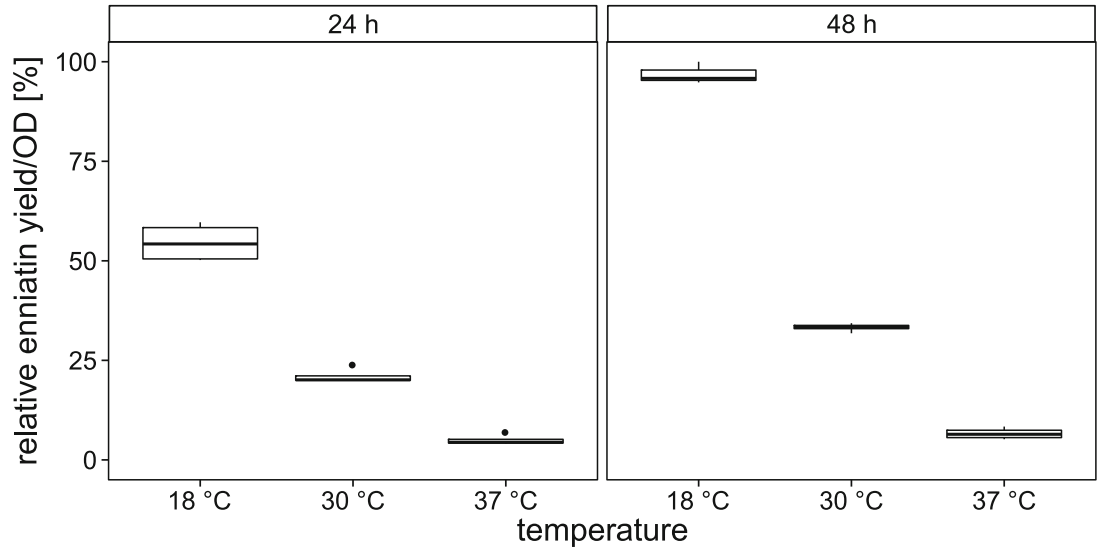

Enniatin production of a Bacillus strain carrying the esyn gene and displaying only a basal synthesis of D-Hiv is dependent on supplementation of this building block to the medium, and this strain is therefore a dependent host. Based on the absence of the native fungal D-hydroxyisovalerate dehydrogenase, which catalyzes the transformation of 2ketoisovalerate (2-Kiv) to D-Hiv, the substrate of ESYN (Lee et al. 1992) is missing in the recombinant host $B$. subtilis. Low production of enniatin was observed without supplementation, indicating that there is some D-Hiv available, probably in the undefined complex media ingredients like yeast extract. Upon addition of various concentrations of D-Hiv, we were able to enhance enniatin production significantly, although the overall feeding of D-Hiv above $5 \mathrm{~mm}$ did not further influence enniatin yields (Fig. 3a). In addition, requirements for $\mathrm{L}-\mathrm{Val}$ in primary metabolism, i.e. ribosomal synthesis of L-Val-rich ESYN (7.8 \%) and enniatin was compensated by feeding LVal. Hence, adding $5 \mathrm{~mm}$ of L-Val to the cultures after induction boosted the enniatin production of BsSZ4 by at least another $10 \%$ as determined by HPLC-MS (Fig. 3b).

In further experiments, we tested the inductor acetoin in different concentrations in the range of $0.1,0.5,1.0$ and $1.5 \%$ $(v / v)$. We observed an increase of 10-20\% enniatin production using 1.0 and $1.5 \%$ acetoin for induction of ESYN expression (Fig. S6). In contrast to the concentration range of 0.1 and $0.5 \%$ final acetoin, higher amounts of acetoin $(1.0$ and $1.5 \%$ ) are coupled to increased cell lysis (data not shown). Therefore, for further experiments and optimization regarding the genetic background of the enniatin producing strain BsSZ4, we chose $0.1 \%$ acetoin, $5 \mathrm{~mm}$ D-Hiv, no additional L-Val (because no stringent necessity for enniatin production) and $48 \mathrm{~h}$ cultivation time at $18{ }^{\circ} \mathrm{C}$.

As mentioned above, the production of enniatin is accompanied by a surfactin and bacillaene synthesis, which is also reflected by a high level of the proteins SrfAA and SrfAB visible in a SDS-PAGE (Fig. S7) and confirmed by tryptic digest of protein bands in LC-ESI-MS ${ }^{2}$ measurements (data not shown). Since synthesis of unwanted protein appears as an additional metabolic burden, we consequently considered inactivation of these clusters by gene deletion, which ultimately could save equivalents of energy like ATP for substrate activation, coenzyme A, precursors, cofactors and Sfp capacity for posttranslational phosphopantetheinylation of multienzymes. In order to optimize growth behaviour, we first
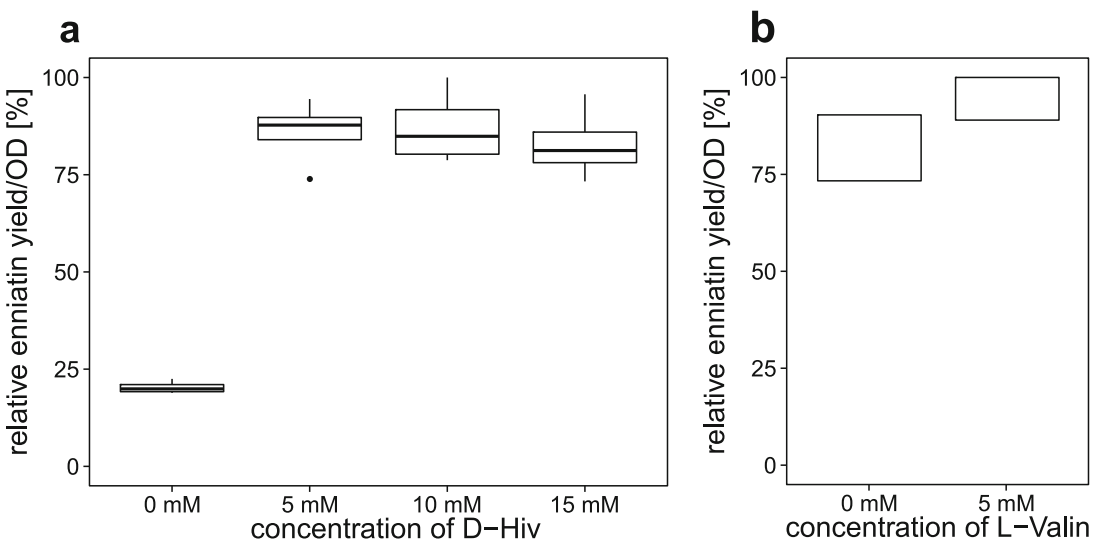

Fig. 3 Influence of precursor feeding on enniatin production. a Supplementation with D-hydroxy isovalerate (D-Hiv): absence of D-Hiv in the medium renders low enniatin yields in the supernatant. A concentration of effect. $N=4$. b Supplementation with L-Val: in order to compensate LVal consumption during protein and peptide synthesis, $5 \mathrm{~mm} \mathrm{~L}$-Val were fed together with $5 \mathrm{~mm}$ D-Hiv to the cultures and raised enniatin production by $>10 \%$. $N=2$; thus, no median is shown 
inactivated genes involved in autolysis $(l y t C)$ and sporulation (spoIIGA). Therefore, we stepwise engineered the BsSZ4 strain with an integrated single copy of esyn and observed a surprisingly significant reduced enniatin synthesis in the resulting BsSZ8 strain (Fig. 4a). An additional inactivation of the surfactin gene cluster in BsSZ10 compensates these losses and leads to an increase of around $20 \%$ of enniatin yield. As opposed to this, an additional inactivation of the bacillaene cluster in BsSZ12 surprisingly led to significantly decreased enniatin production (25\%) compared to BsSZ10.

The genetically modified strains revealed different growth behaviours (Fig. 4b). Particularly the sporulation and $l y t C$ deficient BsSZ8 strain showed decreased optical densities in the time course measurements. The highest $\mathrm{OD}_{600 \mathrm{~nm}}$ of 12.2 was observed for the best enniatin producing strain BsSZ10 being deficient in surfactin synthesis closely followed by the parental strain BsJK28 $\left(\mathrm{OD}_{600 \mathrm{~nm}}=11.4\right)$. The lowest production of enniatin by BsSZ8 (with a lytC/spoIIGA inactivation) correlates with the lowest cell density of $\mathrm{OD}_{600 \mathrm{~nm}}=9.6$ in total. In the wake of BsSZ10 under optimized cultivation conditions together with modification of the genetic background, we obtained a secretory production of $4.7 \mu \mathrm{g} / \mathrm{L}$ enniatin.

We furthermore tested the effect of the cloning of the esyn cluster into the high-copy plasmid pMSE3. The resulting strain BsJK106 (pJK255), which possesses approximately 200 copies of the esyn gene (Fig. S8), produced the highest yield of enniatin (Fig. 5). We quantified the extracts of supernatant of BsJK106 cultures in comparison to an external enniatin standard and measured yields of $1.1 \mathrm{mg} / \mathrm{L}$ enniatin under optimized cultivation conditions. An additional gene copy of the positive transcription factors $\operatorname{sig} L$ and $\operatorname{aco} R$ (Ali et al. 2001; Kabisch et al. 2013b) did not lead to a further enhanced enniatin production (data not shown).

\section{Discussion}

This contribution reports on a cellular system for heterologous expression of a fungal, non-ribosomally synthesized peptide based on the Gram-positive bacterium $B$. subtilis. To assess the suitability of this expression host for heterologous NRPS expression, the non-ribosomal peptide synthetase-encoding gene esyn from the fungus $F$. oxysporum was used. This choice was based on the natural capacity of $B$. subtilis for the overproduction of the non-ribosomally synthesized lipopeptides surfactin and plipastatin or the PKS/NRPShybrid molecule bacillaene. As shown by the results of this study, in B. subtilis, enniatin is actively exported, probably using a native secondary metabolite transporter system, whereas esyn expression in the Gram-negative expression host $E$. coli leads to an exclusive intracellular accumulation of enniatin (data not shown). Similar intracellular accumulation of metabolites was observed in previous studies on heterologous expression in E. coli regarding the homologous cyclooligomer depsipeptide synthetases, e.g. beauvericin synthetase of Beauveria bassiana (Matthes et al. 2012) or valinomycin synthetase produced by Streptomyces tsusimaensis (Jaitzig et al. 2013).

Data of this study show that the cultivation temperature had a significant effect on enniatin production. Lowering the temperature causes decelerated cell growth due to lower metabolic activity, which is a common method to avoid misfolding and aggregation of heterologously expressed proteins (Schein 1989; Vasina and Baneyx 1997). To compensate for the downregulated metabolism at $18{ }^{\circ} \mathrm{C}$, the cultivation duration was doubled, enabling a prolonged and thus increased enniatin production.

In addition, feeding substrates which are building blocks of the enniatin structure boosted enniatin synthesis.
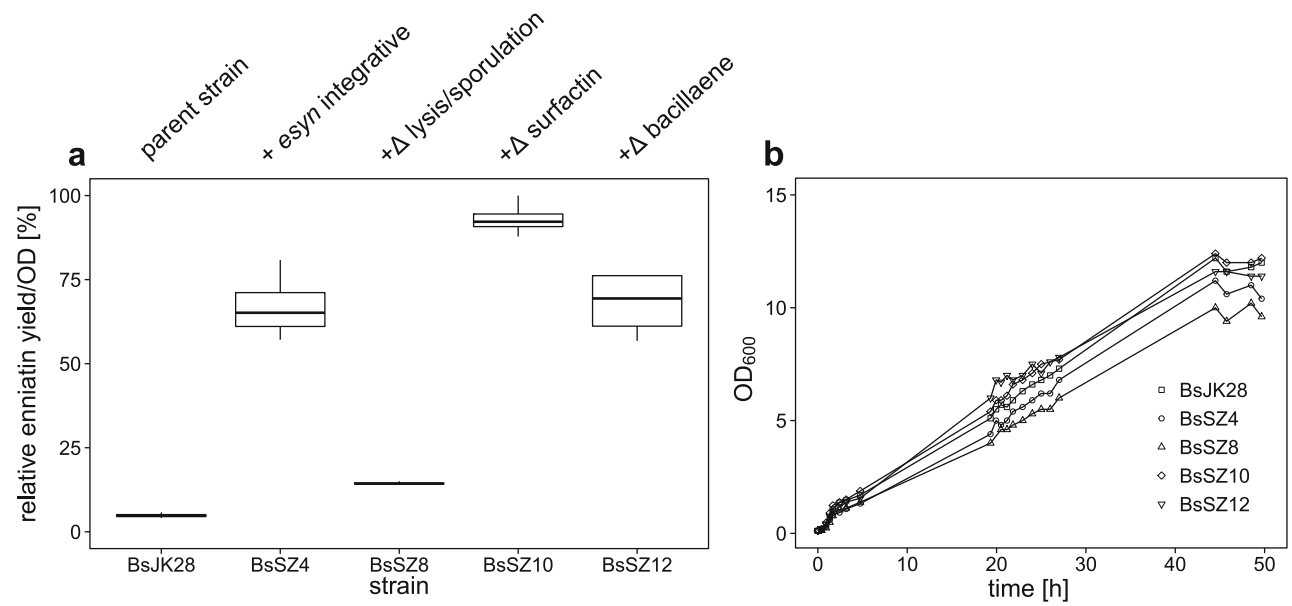

Fig. 4 Engineering of the genetic background of the enniatin producing strain B. subtilis BsSZ4. a Quantitative MRM analysis of secreted enniatin of modified B. subtilis strains after cultivation for $48 \mathrm{~h}$ in SB medium. $N=4$. b Growth curves $\left(\mathrm{OD}_{600 \mathrm{~nm}}\right)$ of engineered B. subtilis

strains during cultivation for $48 \mathrm{~h}$ in SB medium. $N=1$. BsJK28, parental strain; BsSZ4, + esyn; BsSZ8, with a lytC/spoIIGA inactivation; BsSZ10, deletion of the surfactin cluster; BsSZ12, with an additional inactivation of bacillaene synthesis 


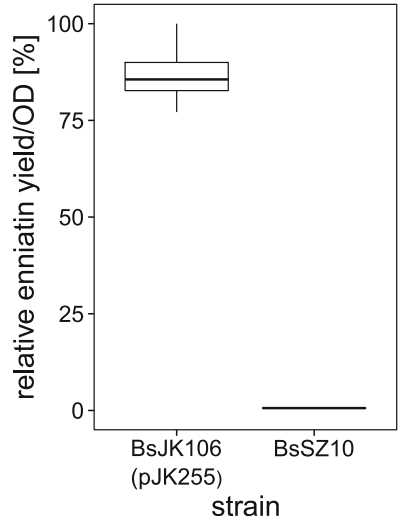

Fig. 5 Extra-chromosomal multi-copy expression of esyn in B. subtilis. Expression of the esyn gene on the multi-copy plasmid pJK255 (with 200 copies) in BsJK106 in comparison to BsSZ10 leads to 130-fold increased enniatin level in the supernatant. $N=4$

Concentrations of $5 \mathrm{mM}$ of L-Val and D-Hiv are sufficient for low expression of the cyclodepsipeptide. Higher feeding amounts of L-Val could promote feedback reactions, which might negatively influence the $\mathrm{L}$-Val biosynthesis regulation as observed for E. coli (Park et al. 2011). Supplementation with $10 \mathrm{~mm}$ up to $15 \mathrm{~mm}$ D-Hiv could also result in oxidation of dispensable D-Hiv through unspecific dehydrogenases from pyruvate and amino acid metabolism, particularly those involved in L-Val, L-Leu and L-Ile biosynthesis (Massey et al. 1976). Excessive addition of L-Val apparently is detrimental and can cause overflow metabolism in the host organism B. subtilis as well as limiting of L-Ile biosynthesis (Castillon et al. 2011; Felice et al. 1977; Leavitt and Umbarger 1962).

For multi-copy expression of esyn, higher concentrations of D-Hiv and L-Val might be required to saturate effective enniatin production. Our data indicate that an increased concentration of the inductor molecule acetoin results in lower cell densities revealing nearly the same amounts of enniatin (data not shown). Reduced cell growth could be caused by higher acetoin concentrations which lead to accumulation of toxic diacetyl, catalyzed by non-enzymatic oxidations (López et al. 1975). Therefore, induction of ESYN expression at $0.1 \%$ acetoin seems to be most advantageous with regard to cell-growth, protein synthesis and coupled enniatin production (Fig. S6).

The negative effect caused by the deletion of the autolysis gene $l y t C$ and the sporulation regulator gene spoIIGA (Kabisch et al. 2013b) on the esyn-expressing strain B. subtilis BsSZ8 was surprising. It could be speculated that the spoIIGA mutation has an effect on the secretion capacity of transporters for secondary metabolites. It is interesting to note that the $\mathrm{ABC}$ transporters OppDF, YtrBE and EcsA are specifically induced during the sporulation process (Leskelä et al. 1999; Perego and Hoch 1996; Yoshida et al. 2000). For YtrBE, a function in the uptake of the inductor acetoin has been suggested. Thus, it is conceivable that a lower intracellular inductor concentration is caused by a potential downregulation of YtrBE in a spoIIGA mutant. Further studies will be required to elucidate the influence of this genetic background on enniatin production in B. subtilis.

However, an additional deletion of the NRPS cluster encoding the surfactin synthetase in strain BsSZ10 complements for above losses in production yields and results in an enhanced enniatin production. On the one hand, the biosynthesis of surfactin relies for a considerable part on the availability of L-Val as a precursor and the capacity for posttranslational modification with Sfp, which could decrease the
Fig. 6 Comparison of the overall optimization of enniatin production in the heterologous producer B. subtilis. Quantitative HPLC-MRM mass spectrometric analysis of crude culture supernatant extracts of BsSZ4 and BsSZ10 as well as BsJK106 shows enhanced enniatin production as a result of a combination of cultivation optimization, genetic modification and high-copy expression of heterologously expressed ESYN

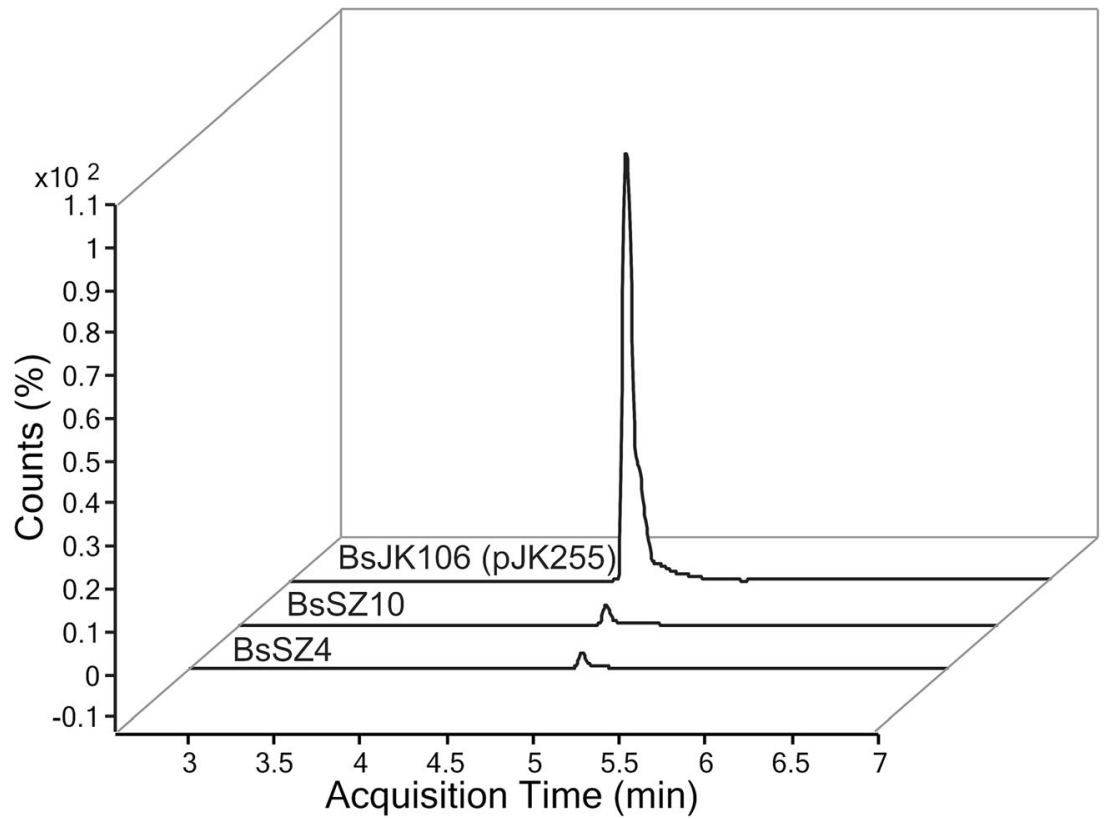


ESYN productivity. Therefore, a deletion of the $s r f A$-cluster might lead to an accumulation of the resources available for enniatin synthesis. On the other hand, this cluster contains a beneficial type II thioesterase SrfAD for regeneration of misprimed nonribosomal peptide synthetase (Schwarzer et al. 2002). Consequently, a co-expression of this type II thioesterase could be advantageous also for the enniatin production. We have addressed not only the gene inactivation of the surfactin-relevant cluster but also the $p k s X$ cluster of the mixed PKS/NRPS secondary metabolite bacillaene (BsSZ12). In this case, the secondary metabolite was identified by means of ESI-mass spectrometry but no high expression level of the synthetase was detected on the protein level in SDS gels (Fig. S7). The slightly negative effect on enniatin concentration after inactivation of the $p k s X$ cluster was quite unexpected (Fig. 4a) and suggests that its inactivation has no direct influence on the availability of precursors or resources of enniatin production.

The highest yield of enniatin secreted into the supernatant was found for strain BsJK106 with the esyn-encoding multicopy plasmid pJK255. This optimized host vector system, which originates from strain BsSZ12 and comprises gene deletions of the $\operatorname{srf} A$ and $p k s X$ clusters, is based on the highcopy plasmid pMSE3. In comparison to other plasmids tested, plasmid pMSE3 is distinguished (Fig. S8) by structural and segregational stability (Silbersack et al. 2006; Swinfield et al. 1991) with 200-250 copies per cell. Despite the metabolic burden of replication and protein synthesis by this high-copy plasmid number, as indicated by lower cell densities, enniatin synthesis significantly increased by $\sim 50 \%$ (Fig. 5). It could be concluded that the deletion of energy- and metabolic precursor-consuming clusters maybe useful for multi-copy expression of ESYN in strain BsJK106. The ultimate strain of this study, BsJK106 (pJK255), produces $1.1 \mathrm{mg} / \mathrm{L}$ enniatin (Fig. 6). We assume that there is further potential for optimization of the secretory enniatin production in $B$. subtilis. This could include classical strain evolvement by mutagenesis as well as the development of suitable fed-batch cultivations including the adjustment of feeding or synthesis of the building blocks D-Hiv and L-Val. First additional optimization steps by overexpressing the appropriate transcription factors AcoR and SigL of the $a c o A$ promoter resulted in an almost complete collapse of enniatin production and cell growth (data not shown). Thus, a balanced ratio of gene copies, transcription and translation regulation as well as concentrations of precursors will be required. For the generation of an autonomous B. subtilis strain producing enniatin without additional D-Hiv feeding, exploitation of the gene coding for the Dhydroxyisovalerate dehydrogenase of $F$. oxysporum (Lee et al. 1992) could be considered by chromosomal integration into strain BsJK106.

To our knowledge, this is the first study to examine the heterologous expression of a NRPS of eukaryotic origin in the
Gram-positive bacterial production host B. subtilis. The approach is considered to have a promising potential being applicable to generate enniatin easily in an economic continuous fermentation process since the entire cyclodepsipeptide is secreted into the medium. Furthermore, this heterologous production of enniatin can also be used for precursor-directed biosynthesis (Feifel et al. 2007; Krause et al. 2001; Matthes et al. 2012; Müller et al. 2009) of new interesting derivatives providing additional or improved bioactivity by feeding and incorporation of $\alpha$-hydroxyl carboxylic acid derivatives.

Acknowledgments The work of S.Z. and R.D.S. was supported by the Cluster of Excellence 'Unifying Concepts of Catalysis' (UniCat) granted by the German Research Council (DFG). The work of J.K. and T.S. was financially supported by the Ministry of Economy, Labour and Tourism of Mecklenburg Vorpommern and the European Fund for Regional Development (V-630-S-177-2013/213; V-630-F-177-2013/212).

Conflict of interest The authors declare that they have no conflict of interest.

Open Access This article is distributed under the terms of the Creative Commons Attribution License which permits any use, distribution, and reproduction in any medium, provided the original author(s) and the source are credited.

\section{References}

Ali NO, Bignon J, Rapoport G, Debarbouille M (2001) Regulation of the acetoin catabolic pathway is controlled by sigma L in Bacillus subtilis. J Bacteriol 183:2497-2504

Arima K, Kakinuma A, Tamura G (1968) Surfactin, a crystalline peptidelipid surfactant produced by Bacillus subtilis: isolation, characterization and its inhibition of fibrin clot formation. Biochem Biophys Res Commun 31:488-494

Billich A, Zocher R (1988) Constitutive expression of enniatin synthetase during fermentative growth of Fusarium scirpi. Appl Environ Microbiol 54:2504-2509

Butcher RA, Schroeder FC, Fischbach MA, Straight PD, Kolter R, Walsh CT, Clardy J (2007) The identification of bacillaene, the product of the PksX megacomplex in Bacillus subtilis. Proc Natl Acad Sci 104: $1506-1509$

Castillon N, Coyle K, Lai J, Yang C (2011) Tetracycline modulates the valine induced stringent response and decreases expressed RpoS in Escherichia coli. JEMI 15:117-124

Chen XH, Koumoutsi A, Scholz R, Eisenreich A, Schneider K, Heinemeyer I, Morgenstern B, Voss B, Hess WR, Reva O, Junge $\mathrm{H}$, Voigt B, Jungblut PR, Vater J, Süssmuth R, Liesegang H, Strittmatter A, Gottschalk G, Borriss R (2007) Comparative analysis of the complete genome sequence of the plant growth-promoting bacterium Bacillus amyloliquefaciens FZB42. Nat Biotechnol 25: $1007-1014$

Dornetshuber R, Heffeter P, Kamyar M-R, Peterbauer T, Berger W, Lemmens-Gruber R (2007) Enniatin exerts p53-dependent cytostatic and p53-independent cytotoxic activities against human cancer cells. Chem Res Toxicol 20:465-473 
Eppelmann K, Doekel S, Marahiel MA (2001) Engineered biosynthesis of the peptide antibiotic bacitracin in the surrogate host Bacillus subtilis. J Biol Chem 276:34824-34831

Feifel SC, Schmiederer T, Hornbogen T, Berg H, Süssmuth RD, Zocher R (2007) In vitro synthesis of new enniatins: probing the $\alpha$-D-hydroxy carboxylic acid binding pocket of the multienzyme enniatin synthetase. ChemBioChem 8:1767-1770

Felice MD, Squires C, Levinthal M, Guardiola J, Lamberti A, Iaccarino M (1977) Growth inhibition of Escherichia coli K12 by L-valine: a consequence of a regulatory pattern. Mol Gen Genet MGG 156:1-7

German-Fattal M (1988) Bactericidal activity of fusafungine towards nosocomial methicillin-resistant staphylococcal strains. Rhinol Suppl 5:29-36

Glinski M, Urbanke C, Hornbogen T, Zocher R (2002) Enniatin synthetase is a monomer with extended structure: evidence for an intramolecular reaction mechanism. Arch Microbiol $178: 267-273$

Jaitzig J, Li J, Süssmuth RD, Neubauer P (2013) Reconstituted biosynthesis of the nonribosomal macrolactone antibiotic valinomycin in Escherichia coli. ACS Synth Biol 3:432-438

Kabisch J, Pratzka I, Meyer H, Albrecht D, Lalk M, Ehrenreich A, Schweder T (2013a) Metabolic engineering of Bacillus subtilis for growth on overflow metabolites. Microb Cell Factories 12:72

Kabisch J, Thürmer A, Hübel T, Popper L, Daniel R, Schweder T (2013b) Characterization and optimization of Bacillus subtilis ATCC 6051 as an expression host. J Biotechnol 163:97-104

Kamyar M, Rawnduzi P, Studenik CR, Kouri K, Lemmens-Gruber R (2004) Investigation of the electrophysiological properties of enniatins. Arch Biochem Biophys 429:215-223

Karam-Sarkis D, German-Fattal M, Bourlioux P (1991) Effect of fusafungine on adherence of Haemophilus influenzae type $\mathrm{b}$ to human epithelial cells in vitro. Biomed Pharmacother 45: 301-306

Kim L, Mogk A, Schumann W (1996) A xylose-inducible Bacillus subtilis integration vector and its application. Gene 181:71-76

Kleinkauf H, von Döhren H (1990) Nonribosomal biosynthesis of peptide antibiotics. Eur J Biochem 192:1-15

Koehn FE, Carter GT (2005) The evolving role of natural products in drug discovery. Nat Rev Drug Discov 4:206-220

Kouri K, Kamyar MR, Lemmens-Gruber R (2003) Actions of the antibiotic mycotoxins beauvericin and enniatin on mammalian tissue. Forum Nutr 56:413-414

Krause M, Lindemann A, Glinski M, Hornbogen T, Bonse G, Jeschke P, Thielking G, Gau W, Kleinkauf H, Zocher R (2001) Directed biosynthesis of new enniatins. J Antibiot (Tokyo) 54:797-804

Kumpfmüller J, Kabisch J, Schweder T (2013) An optimized technique for rapid genome modifications of Bacillus subtilis. J Microbiol Methods 95:350-352

Leavitt RI, Umbarger HE (1962) Isoleucine and valine metabolism in Escherichia coli XI. K-12. J Bacteriol 83:624-630

Lee C, Görisch H, Kleinkauf H, Zocher R (1992) A highly specific D-hydroxyisovalerate dehydrogenase from the enniatin producer Fusarium sambucinum. J Biol Chem 267: 11741-11744

Leskelä S, Wahlström E, Hyyryläinen H-L, Jacobs M, Palva A, Sarvas M, Kontinen VP (1999) Ecs, an ABC transporter of Bacillus subtilis: dual signal transduction functions affecting expression of secreted proteins as well as their secretion. Mol Microbiol 31:533-543

Leuschner RGK, Robinson TP, Hugas M, Cocconcelli PS, RichardForget F, Klein G, Licht TR, Nguyen-The C, Querol A, Richardson M, Suarez JE, Thrane U, Vlak JM, von Wright A (2010) Qualified presumption of safety (QPS): a generic risk assessment approach for biological agents notified to the European Food Safety Authority (EFSA). Trends Food Sci Technol 21:425-435
López JM, Thomas B, Rehbein H (1975) Acetoin degradation in Bacillus subtilis by direct oxidative cleavage. Eur J Biochem 57:425-430

Lund VJ, Grouin JM, Eccles R, Bouter C, Chabolle F (2004) Efficacy of fusafungine in acute rhinopharyngitis: a pooled analysis. Rhinology 42:207-212

Massey LK, Sokatch JR, Conrad RS (1976) Branched-chain amino acid catabolism in bacteria. Bacteriol Rev 40:42-54

Matthes D, Richter L, Müller J, Denisiuk A, Feifel SC, Xu Y, EspinosaArtiles P, Süssmuth RD, Molnár I (2012) In vitro chemoenzymatic and in vivo biocatalytic syntheses of new beauvericin analogues. Chem Commun 48:5674-5676

Middleton R, Hofmeister A (2004) New shuttle vectors for ectopic insertion of genes into Bacillus subtilis. Plasmid 51:238-245

Mongkolthanaruk W (2012) Classification of Bacillus beneficial substances related to plants, humans and animals. J Microbiol Biotechnol 22:1597-1604

Müller J, Feifel SC, Schmiederer T, Zocher R, Süssmuth RD (2009) In vitro synthesis of new cyclodepsipeptides of the PF1022-type: probing the $\alpha$-D-hydroxy acid tolerance of PF1022 synthetase. ChemBioChem 10:323-328

Nakano MM, Magnuson R, Myers A, Curry J, Grossman AD, Zuber P (1991) SrfA is an operon required for surfactin production, competence development, and efficient sporulation in Bacillus subtilis. J Bacteriol 173:1770-1778

Nocon J, Steiger MG, Pfeffer M, Sohn SB, Kim TY, Maurer M, Russmayer H, Pflugl S, Ask M, Haberhauer-Troyer C, Ortmayr K, Hann S, Koellensperger G, Gasser B, Lee SY, Mattanovich D (2014) Model based engineering of Pichia pastoris central metabolism enhances recombinant protein production. Metab Eng 24:129-138

Park JH, Kim TY, Lee KH, Lee SY (2011) Fed-batch culture of Escherichia coli for L-valine production based on in silico flux response analysis. Biotechnol Bioeng 108:934-946

Patel PS, Huang S, Fisher S, Pirnik D, Aklonis C, Dean L, Meyers E, Fernandes P, Mayerl F (1995) Bacillaene, a novel inhibitor of procaryotic protein synthesis produced by Bacillus subtilis: production, taxonomy, isolation, physico-chemical characterization and biological activity. J Antibiot (Tokyo) 48:997-1003

Perego M, Hoch JA (1996) Cell-cell communication regulates the effects of protein aspartate phosphatases on the phosphorelay controlling development in Bacillus subtilis. Proc Natl Acad Sci 93:1549-1553

Petsch D, Anspach FB (2000) Endotoxin removal from protein solutions. J Biotechnol 76:97-119

Pfeifer BA, Khosla C (2001) Biosynthesis of polyketides in heterologous hosts. Microbiol Mol Biol Rev 65:106-118

Pieper R, Haese A, Schröder W, Zocher R (1995) Arrangement of catalytic sites in the multifunctional enzyme enniatin synthetase. Eur J Biochem 230:119-126

Pleiss U, Turberg A, Harder A, Londershausen M, Jeschke P, Boheim G (1996) Synthesis of a radiolabeled enniatin cyclodepsipeptide [3Hmethyl]JES 1798. J Label Compd Radiopharm 38:651-659

Samant S, Gupta G, Karthikeyan S, Haq SF, Nair A, Sambasivam G, Sukumaran S (2014) Effect of codon-optimized E. coli signal peptides on recombinant Bacillus stearothermophilus maltogenic amylase periplasmic localization, yield and activity. J Ind Microbiol Biotechnol 41:1435-1442

Schein CH (1989) Production of soluble recombinant proteins in bacteria. Nat Biotechnol 7:1141-1149

Schwarzer D, Mootz HD, Linne U, Marahiel MA (2002) Regeneration of misprimed nonribosomal peptide synthetases by type II thioesterases. Proc Natl Acad Sci 99:14083-14088

Sietske de Boer A, Diderichsen B (1991) On the safety of Bacillus subtilis and B. amyloliquefaciens: a review. Appl Microbiol Biotechnol 36: $1-4$

Silbersack J, Jürgen B, Hecker M, Schneidinger B, Schmuck R, Schweder T (2006) An acetoin-regulated expression system of Bacillus subtilis. Appl Microbiol Biotechnol 73:895-903 
Stein T (2005) Bacillus subtilis antibiotics: structures, syntheses and specific functions. Mol Microbiol 56:845-857

Süssmuth R, Müller J, von Döhren H, Molnár I (2011) Fungal cyclooligomer depsipeptides: from classical biochemistry to combinatorial biosynthesis. Nat Prod Rep 28:99

Swinfield TJ, Jannière L, Ehrlich SD, Minton NP (1991) Characterization of a region of the Enterococcus faecalis plasmid pAM beta 1 which enhances the segregational stability of pAM beta 1-derived cloning vectors in Bacillus subtilis. Plasmid 26:209-221

Tsuge K, Matsui K, Itaya M (2007) Production of the non-ribosomal peptide plipastatin in Bacillus subtilis regulated by three relevant gene blocks assembled in a single movable DNA segment. J Biotechnol 129:592-603

Vasina JA, Baneyx F (1997) Expression of aggregation-prone recombinant proteins at low temperatures: a comparative study of the Escherichia coli cspA and tac promoter systems. Protein Expr Purif 9:211-218
Wickham H (2009) ggplot2: elegant graphics for data analysis. Springer, New York

Yan X, Yu H-J, Hong Q, Li S-P (2008) Cre/lox system and PCR-based genome engineering in Bacillus subtilis. Appl Environ Microbiol 74:5556-5562

Yoshida K-I, Fujita Y, Ehrlich SD (2000) An operon for a putative ATPbinding cassette transport system involved in acetoin utilization of Bacillus subtilis. J Bacteriol 182:5454-5461

Zeigler DR, Pragai Z, Rodriguez S, Chevreux B, Muffler A, Albert T, Bai R, Wyss M, Perkins JB (2008) The origins of 168, W23, and other Bacillus subtilis legacy strains. J Bacteriol 190:6983-6995

Zhang Y, Buchholz F, Muyrers JP, Stewart AF (1998) A new logic for DNA engineering using recombination in Escherichia coli. Nat Genet 20:123-128

Zocher R, Keller U, Kleinkauf H (1982) Enniatin synthetase, a novel type of multifunctional enzyme catalyzing depsipeptide synthesis in Fusarium oxysporum. Biochemistry (Mosc) 21: $43-48$ 